Original Research

\title{
Zinc Silicate Thin Film Composites Obtained by a Sputtering Based Approach: Structural, Dielectric and Photovoltaic Properties
}

Anna Castaldo *, Emilia Gambale, Giuseppe Vitiello

ENEA, TERIN-STSN-SCIS, Piazzale Enrico Fermi, 1, Portici, ITALY; E-Mails: anna.castaldo@enea.it; emilia.gambale@enea.it; giuseppe.vitiello@enea.it

* Correspondence: Anna Castaldo; E-Mail: anna.castaldo@enea.it

Academic Editor: Joaquin Alonso-Montesinos

Special Issue: Photovoltaic Solar Systems and Solar Thermal Plants

Journal of Energy and Power Technology

2021, volume 3, issue 2

doi:10.21926/jept.2102015
Received: January 19, 2021

Accepted: March 22, 2021

Published: April 02, 2021

\begin{abstract}
This study deals with the production of zinc silicate thin films employing a solid-state reaction at temperatures ranging from $300{ }^{\circ} \mathrm{C}$ to $560{ }^{\circ} \mathrm{C}$, to develop new inorganic, $\mathrm{n}$-type materials with seemingly incompatible properties as low work function, high mobility, and high visible transmittance. Depending on a careful reaction control, zinc silicates can preserve reagents (silicon and/or $\mathrm{ZnO}$ ) as nanoaggregate guests, exhibiting tunable light absorption and emission properties that make them useful for different applications, e.g., LED phosphors, photovoltaic windows, mesoporous photocatalytic materials for solar fuel production, etc. The main aim of this work is to obtain a zinc silicate single-phase and then determine its dielectric properties through ellipsometry. Simultaneously, as an example of application, a p$\mathrm{n}$ photovoltaic junction is fabricated that exhibits a remarkable collection of photo-generated charge carriers at very high bandgaps $(>2 \mathrm{eV})$. The obtained results are promising in tailoring the zinc silicate guest-host composition (appropriate Si nanostructures sizing and quantity, improved charge carrier transport properties due to the simultaneous presence of bulk zinc oxide islands) in the function of the desired application, and taking into account that proposed selected low-temperature fabrication process is strategic for safeguarding the underlying junctions of tandem devices.
\end{abstract}

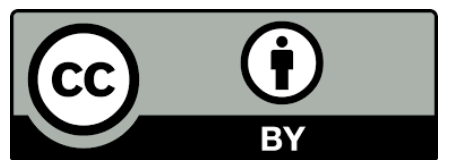

(C) 2021 by the author. This is an open access article distributed under the conditions of the Creative Commons by Attribution License, which permits unrestricted use, distribution, and reproduction in any medium or format, provided the original work is correctly cited. 


\section{Keywords}

Zinc silicate; sputtering; guest host inorganic composites; silicon nanocrystals; zinc oxide; complex refractive index; dielectric properties; photovoltaics; $p$-n junction; photo-generated carriers

\section{Introduction}

Zinc silicates having good chemical stability, low cost, high visible transmittance, luminescence properties, tunable dielectric constants, and mesoporous structures have received considerable attention in the recent literature for different applications like catalysts [1], optoelectronic devices [2], high k-dielectric nanoscale transistors [3], phosphors [4], innovative matrices for dispersing silicon nanostructures [5], etc. The characteristics of n-type materials with low work function, high mobility, and eminently visible transmittance, apparently incompatible, make them ideal for developing novel and interesting metamaterials with tailorable NLO properties [6].

Practically, several zinc silicates exist in amorphous and crystalline phases: orthorhombic, monoclinic, and rhombohedral (corundum type) phases like zinc meta-silicate, $\mathrm{ZnSiO}_{3}$, and rhombohedral, tetragonal, and cubic (spinel-type) phases like zinc orthosilicate, $\mathrm{Zn}_{2} \mathrm{SiO}_{4}$ [7]. The most diffused and utilized ones are willemite and $\mathrm{Zn}_{2} \mathrm{SiO}_{4}$, extracted by the homonym mineral and the pyroxene-like compound of $\mathrm{ZnSiO}_{3}$ [8]. Different synthetic procedures for zinc silicates are known: sintering from the $\mathrm{ZnO}$ and $\mathrm{SiO}$ powders [9], the hydrothermal method [10], spray pyrolysis [11], sol-gel method [12], etc., and they often require high temperatures.

In a previous work [13], we described an original approach based on a simple chemical reaction in a vacuum between sputtered zinc oxide and amorphous silicon reagents for dispersing silicon quantum dots (Si-QDs) in an amorphous matrix of zinc silicates. The overall process can be regarded as innovative because it was performed at a comparatively low temperature, around $500{ }^{\circ} \mathrm{C}$ (the typical temperature for producing silicon nanostructures is higher than $1000^{\circ} \mathrm{C}$ [14]). The thickness of a sputtered thin layer of $\mathrm{Si}$ and $\mathrm{ZnO}$, annealing temperature, and annealing duration strongly affect the reaction mechanism and, as a consequence, the final products that are zinc silicates, $\mathrm{Si}$ QDs, ZnO nanocrystals. The peculiar properties, such as quantum confinement, luminescence emission, and electric tunneling, of the silicate dielectric matrix, guesting Si-QDs, are bestowed by the guest [15].

Here, we deepen and enlarge the said approach with further control of the process and dielectric characterization of the zinc silicate thin film. The main aim of this work is to obtain zinc silicate single-phase as a product to study its dielectric properties. In particular, using UV-Vis-NIR and ellipsometric analysis, the dielectric constant, refractive index, and optical bandgap of such matrix are determined. The second aim is to produce composites with high electrical conductivity by combining them whit a $p$-type material and verifying if the resulting $p-n$ junction exhibits photovoltaic properties. 


\section{Materials and Methods}

We synthesized multi-layered Si-ZnO structures by magnetron sputtering technique, using a planar magnetron multi-cathode sputtering apparatus by Material Research Corporation Mod, MRC 643. Boron doped $\mathrm{Si}$ and undoped $\mathrm{ZnO}$ sputter targets (dimensions: $\mathrm{H}=38 \mathrm{~cm}$ and $\mathrm{L}=12 \mathrm{~cm}$ ) were used, coupled with bi-polar pulsed DC and RF generator, respectively. Quartz sheets, borosilicate glasses, and silicon wafer $<111>$ were used as substrates. Sputtering depositions were carried out at pressures ranging from 0.53 to $2.0 \mathrm{~Pa}$, starting from a base vacuum of about $2 \times 10^{-4} \mathrm{~Pa}$, with variable powers supplied to the targets of silicon and zinc oxide, comprised in the range 350-450 W and $700-1000 \mathrm{~W}$, respectively.

The different sputtered $\mathrm{Si}-\mathrm{ZnO}$ samples were utilized as reagents for the solid-state reaction conducted in air or inside a programmable oven operating at a vacuum of $2 \times 10^{-2} \mathrm{~Pa}$ and temperatures ranging from $300{ }^{\circ} \mathrm{C}$ to $560{ }^{\circ} \mathrm{C}$. The molar ratio of $\mathrm{Zn} / \mathrm{Si}$ was determined from the deposition rate and thicknesses of single material films deposited in all conditions. The annealing duration $\left(t_{a n n}\right)$ ranged from 3 to $18 \mathrm{~h}$ in air or vacuum, with the criterion of prolonging until the disappearance of reagents for obtaining thin zinc silicates samples or interrupting after 3-6 $\mathrm{h}$ for preserving reagents as silicon nanostructures and bulk zinc oxide islands or nanocrystals. A large number of samples were produced and analyzed, starting from different initial $\mathrm{Zn} / \mathrm{Si}$ molar ratios and stopping the reaction at different times. The thickness of the sputtered samples was measured using a Tencor P-10 surface profiler. Ultraviolet-Visible-Near Infrared (UV-VIS-NIR) reflectance and transmittance measurements were carried out in the range 250-2000 nm utilizing a Perkin Elmer Lambda 900 double beam spectrophotometer, and the spectral absorption coefficient $\alpha(\lambda)$ was calculated. Scanning TEM (STEM) and High-Resolution TEM (HRTEM) images were recorded using a JEOL JEM 2010F TEM operating at $200 \mathrm{kV}$ accelerating voltage and equipped with a field emission gun. The dielectric constants, refractive index $n$, and extinction coefficient $k$ of zinc silicates were determined by mathematical inversion of the experimental reflectance $(R)$ and transmittance $(T)$ data. The model of the structure used for the mathematical inversion is presented in Figure 1 . The total reflectance and the total transmittance of the structure are given by the Fresnel equations as follows:

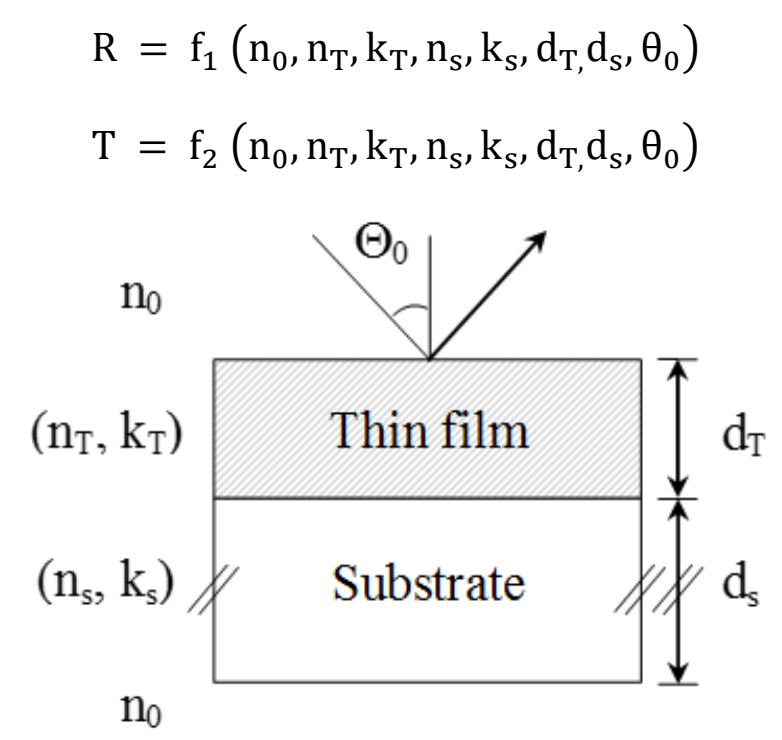

Figure 1 Model used for the mathematical inversion procedure. 
Here, $n_{0}$ is the refractive index of air, $n_{T}$, and $k_{T}$ are the refractive index and extinction coefficient of the thin film, $n_{s}$ and $k_{s}$ are those of the glass substrate, $d_{T}$ and $d_{S}$ are the thicknesses of the thin film and substrate, respectively, and $\theta_{0}$ is the angle of incidence of light on the sample during the measurements. Equation (1) is solved to find $n_{T}$ and $k_{T}$. A linear regression method, based on the interior-reflective Newton algorithm $[16,17]$, was employed to extract $n$ and $k$ of the thin film layer.

For fabricating $\mathrm{p}-\mathrm{n}$ junctions, doped silicon layers were grown on QD-based composites by conventional RF PECVD technique (13.56 MHz), using diborane as a dopant gas to prepare p-type material. X-ray diffraction (XRD) analysis was performed by a Philips X'Pert PROMRD diffractometer working with CuKa radiation $(\lambda=0.154056 \mathrm{~nm})$ at a grazing incidence angle of $0.3^{\circ}$. A $2 \theta$ scan range of $10-95^{\circ}$ was used. For XRD, diffraction peaks matching standard values from the International Centre for Diffraction Data ${ }^{\circledR}\left(\right.$ ICDD $^{\circledR}$, the crystallographic database specifically designed for material identification and characterization) were utilized. Raman scattering measurements were performed at room temperature on a micro-Raman Renishaw system model using an excitation source of 514 $\mathrm{nm}$ of an Argon ion laser at normal incidence. The nominal laser power used was $20 \mathrm{~mW}$. Fourier Transform Infrared (FTIR) spectra were recorded on a Bruker Fourier transform spectrometer model Equinox55 equipped with a DTGS detector operating in the range $400-4000 \mathrm{~cm}^{-1}$. Its resolution was $4 \mathrm{~cm}^{-1}$. The resistivity was measured with a NAPSON four-point probe apparatus (Resitest RT-8A). The electrical properties were obtained by Hall measurement under Van der Pauw configuration at room temperature. Light I-V characteristics of the solar cells were measured at AM 1.5 Global under a solar simulator. Furthermore, the Quantum Efficiency (QE) measurements were performed to get detailed information on the photocurrent generation characteristics of the solar cells. The photogenerated short-circuit current $\left(\mathrm{J}_{\mathrm{sc}}\right)$ was evaluated in the wavelength range of $350-800 \mathrm{~nm}$ by $\mathrm{QE}$ measurements on devices.

\section{Results and Discussion}

A solid-state reaction between stratified silicon and zinc oxide reagents, deposited in different amounts by means of radio-frequency sputtering at a temperature of $450{ }^{\circ} \mathrm{C}$ in a vacuum for $6 \mathrm{~h}$ resulted in zinc silicate thin-film composites. The opaque silicon-like texture of the reagents became transparent or light yellow after the growth, depending on the exact composition of the reaction products after thermal treatment.

A high annealing temperature and/or duration (from 6 to $18 \mathrm{~h}$ ), combined with thin sputtered Si and $\mathrm{ZnO}$ layers, resulted in the formation of transparent zinc silicates. Moreover, low annealing temperature ranging from $300{ }^{\circ} \mathrm{C}$ and/or short annealing duration (from 3 to $6 \mathrm{~h}$ ) and/or thick sputtered $\mathrm{Si}$ and $\mathrm{ZnO}$ layers also resulted in a composite containing non-reacted $\mathrm{Si}$ and/or $\mathrm{ZnO}$ as nanoaggregates, dispersed in the matrix.

We pursued two goals in this analytical work: 1) obtaining a one phase system comprising amorphous zinc orthosilicate to investigate its dielectric properties by means of ellipsometric measurements (that can be better conducted on a homogeneous sample); 2) demonstrating the photovoltaic properties of guest-host n-type inorganic composites (having zinc silicate as host matrix, silicon nanostructures as photoactive guests dispersed inside and natural n-type zinc oxide to improve carrier transport) in a p-n junction.

To reach these goals, different composites were fabricated and characterized by FTIR, UV-VISNIR spectroscopy, micro-RAMAN, XRD, TEM, and ellipsometry. To illustrate the analytical method, 
a generic composite was obtained at a $\mathrm{Zn} / \mathrm{Si}$ molar ratio of 1.48 , by means of an incomplete reaction conducted at $450{ }^{\circ} \mathrm{C}$ in a vacuum for $8 \mathrm{~h}$, and hence reagents containing different phases were utilized.

The first confirmation of zinc silicate formation was obtained from the FTIR transmittance spectra. The shift of peak related to Si-O stretching from the typical value $\left(1100 \mathrm{~cm}^{-1}\right)$ characterizing stoichiometric silica [18] toward lower values $\left(1060 \mathrm{~cm}^{-1}\right)$ was observed. This peak shift, coupled with the presence of broadband at $930-940 \mathrm{~cm}^{-1}$, is attributable to $\mathrm{Si}-\mathrm{O}(-\mathrm{Zn})$ stretching [19] of both zinc orthosilicate $\mathrm{Zn}_{2} \mathrm{SiO}_{4}$ and metasilicate $\mathrm{ZnSiO}_{3}$. Their amorphous nature was confirmed by XRD analysis.

The second analytical step was laid to verify the presence of unreacted silicon and zinc oxide in composites. Clear evidence of nano-crystalline guests came from the micro-Raman, XRD, and TEM analyses.

In Figure 2, the Raman spectrum of a composite obtained by an incomplete reaction conducted at $450{ }^{\circ} \mathrm{C}$ in vacuum for $6 \mathrm{~h}$ and hence containing different phases is reported, as an example of the analytical method utilized for all produced composites.
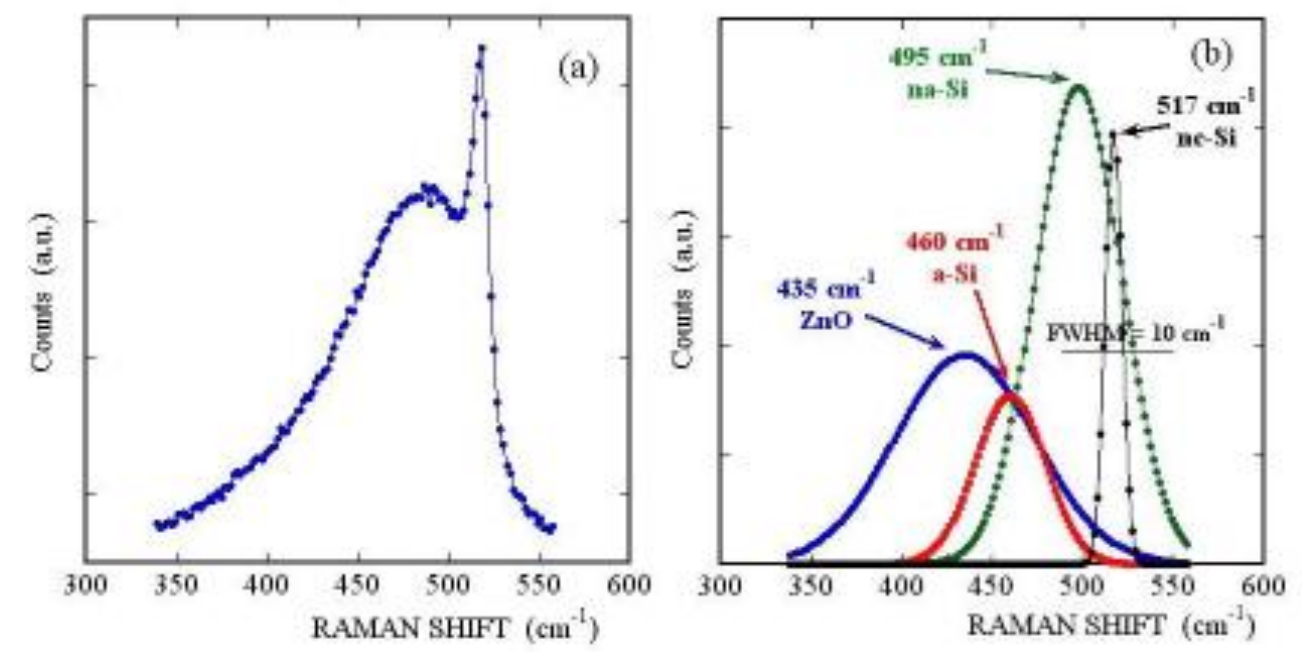

Figure 2 (a) Raman spectrum related to a typical composite from an incomplete reaction.

(b) Deconvolution of the Raman spectrum identifying three phases of silicon (amorphous, nano-amorphous, selected, and nano-crystalline). Peak attributable to ZnO is also well identified.

In the region 350-550 $\mathrm{cm}^{-1}$, the characteristic peak of the transverse optic one photon (TO) mode of crystalline silicon ( $520 \mathrm{~cm}^{-1}$ in bulk c-Si) shifts to lower Raman values depending on the decreasing size of crystallites. In this particular case, it is at $517 \mathrm{~cm}^{-1}$. The shift in Raman peaks was analyzed by a deconvolution approach of the spectrum because peaks are also expected from $E_{2}(T)$ mode of zinc oxide (normally in bulk crystalline $\mathrm{ZnO}$ positioned at $438 \mathrm{~cm}^{-1}$ ) and amorphous silicon in the same spectral region. In particular, a fit of spectral data with four Gaussians gives information on the different phases (see Figure 2), attributed to amorphous $\left(460 \mathrm{~cm}^{-1}\right)$, nano-amorphous $\left(495 \mathrm{~cm}^{-1}\right)$, nano-crystalline $\left(517 \mathrm{~cm}^{-1}\right)$, silicon phases, and $\mathrm{ZnO}$ phase $\left(435 \mathrm{~cm}^{-1}\right)$.

Further evidence on the presence of Si nanocrystals comes from XRD analysis reported in Figure 3. 


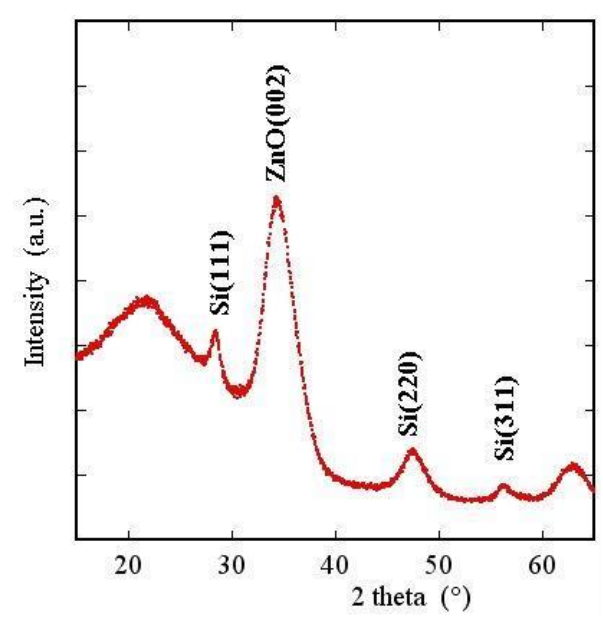

Figure 3 XRD spectrum related to the composite. Peaks attributable to crystalline Si and ZnO appear evidently.

All the XRD peaks were indexed and found to be in agreement with those of the crystalline phase of Silicon (JCPDS card file no. 772111) and with the hexagonal wurtzite phase of ZnO (JCPDS card file no. 043-0002). The sample crystallite size (D) was calculated using Debye-Scherer's equation (2).

$$
D=\frac{0.9 \lambda}{\beta \cos \theta}
$$

Here, $\lambda$ is the wavelength of the $X$-ray beam $(\lambda=1.5406 \AA), \beta$ is the full width at half maximum (FWHM) of the intense peak, and $\theta$ is the Braggs angle. Based on the above calculation, the crystallite size of Si is about $5 \mathrm{~nm}$.

The third analytical step was to define the phases constituting the composite. As such, scope optical bandgaps were defined by UV-VIS-NIR reflectance and transmittance measurements by Tauc plot method, assuming zinc silicates with direct transition, whereas indirect transition was assumed for amorphous and for crystalline Si [20].

In Figure 4 Tauc plots of the composite containing all possible phases are reported as an example of the method.
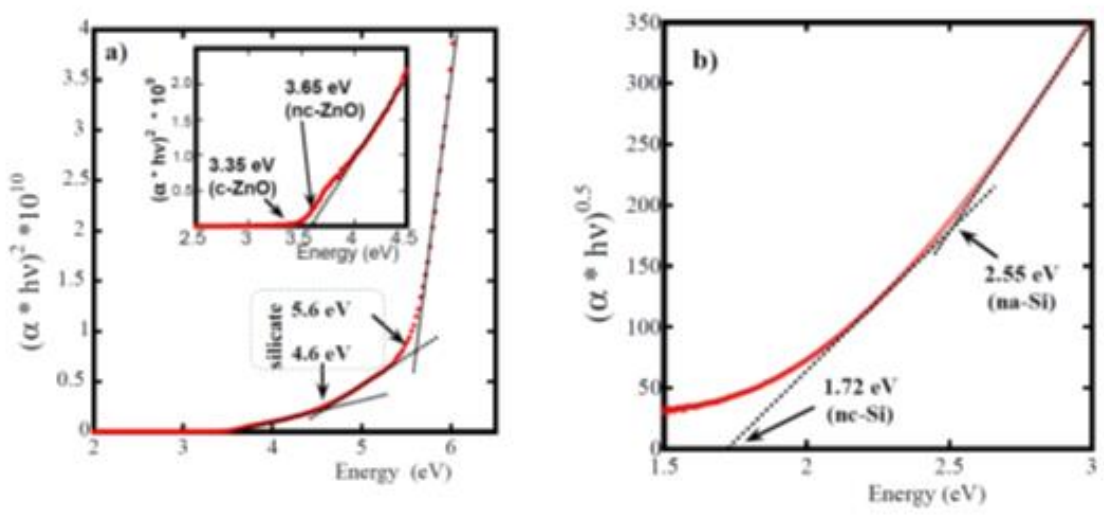

Figure 4 Tauc plot method applied to a composite containing different phases. Optical bandgap values correlated to phases with a) direct transition (zinc oxide and silicates); b) indirect transition (amorphous and crystalline silicon). 
In particular, in Figure 4a, two distinct linear regions can be observed, and the presence of two different zinc silicates (ortho and meta) with similar bandgap values of $5.6 \mathrm{eV}$ and $4.6 \mathrm{eV}$ can be confirmed. Silicon nanocrystals are present with bandgap value of $1.72 \mathrm{eV}$. Amorphous silicon nanoparticles were identified by their bandgap value of $2.55 \mathrm{eV}$. $\mathrm{ZnO}$ in bulk form and as nanocrystals were identified by bandgap values of $3.35 \mathrm{eV}$ and $3.65 \mathrm{eV}$, respectively. The presence of $\mathrm{ZnO}$ phases could be justified by the hypothesis that $\mathrm{ZnO}$ sputtered layers are not entirely consumed during the thermally activated reaction, silicon being the limiting reagent $(\mathrm{Zn} / \mathrm{Si} \mathrm{mol} / \mathrm{mol}>$ 1 in the sputtered reagents). Hence, islands of bulk zinc oxide are found in some composites, while in some others, only ZnO nanocrystals are found.

The cross-sectional images of the composite, obtained by transmission electron microscopy, $\mathrm{X}$ TEM, at an acceleration voltage of $200 \mathrm{kV}$, are reported in Figure 5 . The study of the images of the film shows silicon and zinc oxide nanocrystals embedded in a heterogeneous silicate matrix with alternate regions in terms of different brightness, correlated to the presence (dark regions) or absence (clear regions) of Zn-O-Si bond. For nanocrystals identification, an interplanar spacing of $19.7 \mathrm{~nm}$ for nanoaggregate, in the bright region, is very close to the value of $19.2 \mathrm{~nm}$ of the lattice parameter in (220) direction of the crystalline silicon structure; an interplanar spacing of $25.7 \mathrm{~nm}$ is very close to $26.06 \mathrm{~nm}$ of the lattice parameter in (002) direction of the $\mathrm{ZnO}$ (wurtzite) phase. Both the types of nanocrystals have spherical and ellipsoidal shapes, with the smallest dimension of the grain ranging from 4 to $7 \mathrm{~nm}$. Si Plasmon image reported in Figure 4c was obtained by Energy Filtered Transmission Electron Microscopy, EFTEM, an imaging technique exploiting the inelastic scattered electrons. From this picture, the stratified lamellar distribution of silicon inside the composite becomes evident.
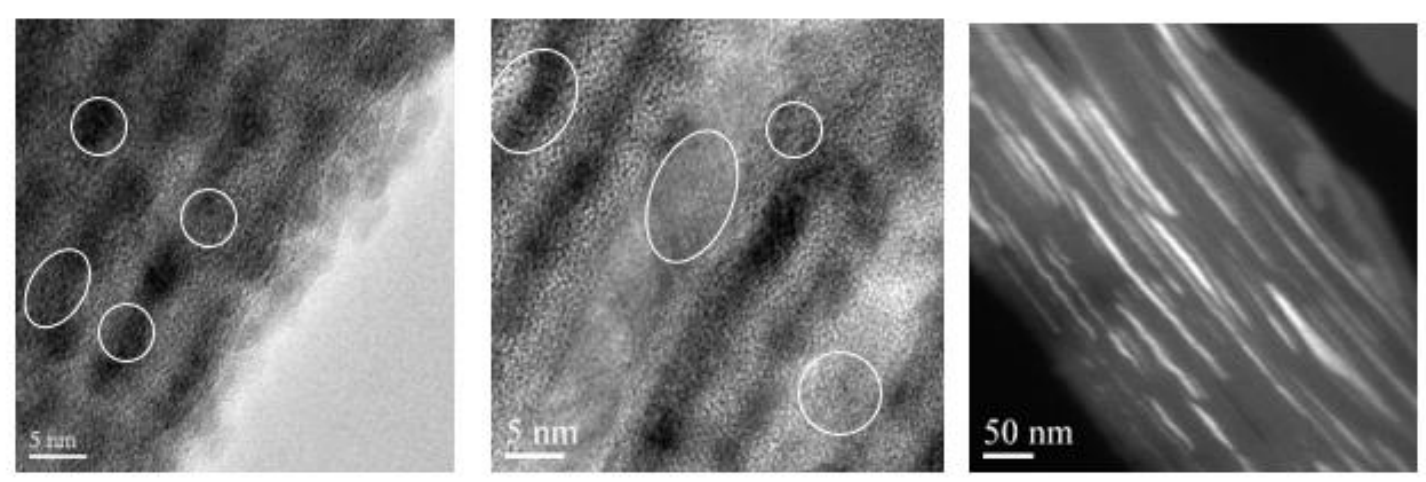

Figure 5 HRTEM images of a zinc silicate composite containing Silicon (a) and Zinc oxide nanocrystals (b). EFTEM image, the bright regions depict silicon (c).

The results obtained by TEM observation on composites can be illustrated in a schematic picture (see Figure 6) where Si nano-crystalline and nanoamorphous (nc-Si, na-Si) and ZnO-nanocrystals (nc$\mathrm{ZnO}$ ) are embedded in a hetero-superlattice (alternate layers of silicate rich of Si-O-Si or $\mathrm{Zn}-\mathrm{O}-\mathrm{Zn}$ bonds). 


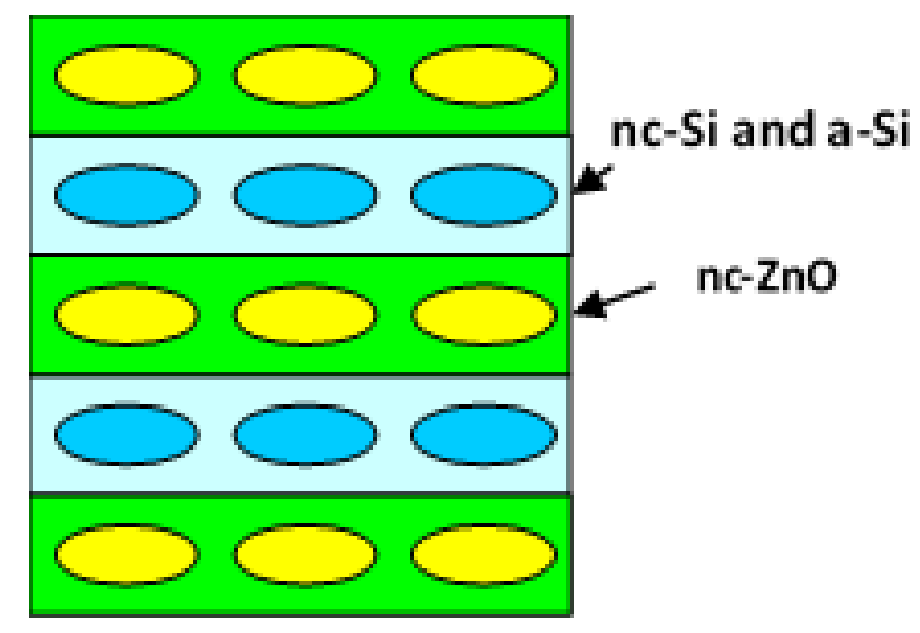

Figure 6 Scheme of the solid-state reaction products. Light blue regions are Si-O-Si rich domains and contain nc-Si and nano a-Si; green regions are $\mathrm{Zn}-\mathrm{O}-\mathrm{Zn}$ rich domains and contain nc-ZnO.

Considering the stratified structure of the composites, it is reasonable to have anisotropy in the electrical resistivity. In fact, on performing resistivity measurements in planar and sandwich configurations, the values found are very different for the composite under analysis. The values are $16 \Omega \mathrm{cm}$ and $4.7 \times 10^{4} \Omega \mathrm{cm}$, respectively.

Repeating all the analytical steps for many samples, corresponding to different initial $\mathrm{Zn} / \mathrm{Si}$ molar ratios and various experimental conditions (in terms of time, temperature, environment, and so on), from the overall characterization, the dataset suggests that it is possible to obtain a one phase insulating, amorphous, and transparent $\mathrm{Zn}_{2} \mathrm{SiO}_{4}$ composite with a comparatively low bandgap ( $<5.6$ $\mathrm{eV}$ ), when the reaction is complete (annealing in the air for a long time starting from a stoichiometric reagents ratio). Such a matrix is a promising alternative to silica (like as $\mathrm{Si}_{3} \mathrm{~N}_{4}$ or $\mathrm{SiC}$ in Conibeer works [21]) because decreasing barrier height (from $9 \mathrm{eV}$ of silica to $5.5 \mathrm{eV}$ ) increases the tunneling probability between adjacent silicon nanostructures, whose eventual presence can be promoted when the reaction is incomplete. By appropriate variation in the power supplied to the sputtering targets for the reagent deposition and in thermal annealing conditions (time, duration, vacuum conditions), a convenient window for obtaining conductive composites is found, where hopping mechanism between the tailored (in terms of grain size, composition, and quantity) Si nanostructures through zinc silicate thin layers can be hypothesized. Moreover, the simultaneous presence of unreacted conductive zinc oxide thin layers (crystalline phase with an optical bandgap of $3.2 \mathrm{eV}$ ) gives a quasi-metallic transport mechanism to composites.

For performing dielectric characterization of the matrix, a thin film material named C100-45 was selected containing a single phase of insulating zinc o-silicate, which was obtained choosing 1.67 as reagent molar ratio and relatively long annealing time $(12 \mathrm{~h})$ in air. Simultaneously, by choosing a $\mathrm{Zn} / \mathrm{Si}$ molar ratio of 1.32 and a sufficiently low annealing temperature $\left(450{ }^{\circ} \mathrm{C}\right)$ in vacuum, two composites, namely A85-45 and B100-45, were produced for optimizing electrical properties and fabricating the $n$-type thin film for a $p$-n demonstrative junction.

Table 1 reports the composition in terms of the presence of different phases (determined by evaluating optical bandgaps) of the three samples selected for applications. 
Table 1 Thin films chemical compositions.

\begin{tabular}{llllllll}
\hline Sample & $\begin{array}{c}\text { Zn/Si ratio } \\
\text { (mol/mol) }\end{array}$ & O-Silicate & M-Silicate & Na-Si & Nc-Si & c-ZnO & NcZnO \\
& & & & & & & \\
\hline A85-45 & 1.32 & 5.5 & 4.4 & 2.80 & 2.00 & 3.32 & - \\
B100-45 & 1.32 & 5.5 & 4.3 & 2.53 & 1.90 & 3.38 & - \\
C100-45 & 1.67 & 5.5 & - & - & - & - & - \\
\hline
\end{tabular}

Sample C100-45 was analyzed using ellipsometry (Cfr Figure 6). The refractive index of the thin film is in the range of 1.606-1.472 at wavelengths between 250 and $2000 \mathrm{~nm}$. The dielectric constant is in the range 2.16-2.60 at wavelengths between 250 and $2000 \mathrm{~nm}$. This result makes such matrix an interesting candidate for the ultralow dielectric constant (low-k) thin-film material, considering that the traditional $\mathrm{SiO}_{2}$ has a dielectric constant of around 4 .
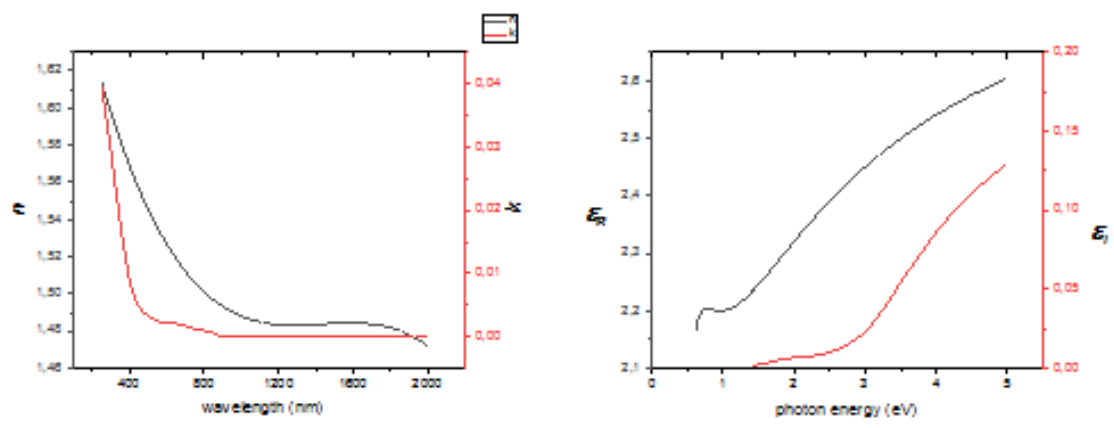

Figure 7 Complex Refractive index (a) and dielectric constant (b) v/s wavelength of the sample C100-45.

As concerned with the feasibility of a photovoltaic junction using composites A85-45 and B10045 , the topic has been addressed in a separate paragraph.

\subsection{Photovoltaic p-n Devices}

The Hall measurements of the composites indicated the n-type behavior with carrier concentrations ranging from $10^{16}$ to $10^{17} \mathrm{~cm}^{-3}$. Two $\mathrm{p}$-n devices were fabricated with the following deposition sequence (see Figure 8): (i) multi-layered Si-ZnO materials of 0.28 and $0.57 \mu \mathrm{m}$ thickness, namely, A85-45 and B100-45, respectively, were deposited on tungsten-coated glass and subsequently annealed for $8 \mathrm{~h}$ at $450^{\circ} \mathrm{C}$ under vacuum; (ii) on the previous structures, a $30 \mathrm{~nm}$ thick boron-doped c-Si layer was deposited by PECVD technique; (iii) ZnO: Al circular front contacts with a diameter of $0.5 \mathrm{~cm}$ were deposited by sputtering technique and a thickness of $0.5 \mu \mathrm{m}$ and sheet resistance of $15 \Omega / \mathrm{sq}$. These two devices were named as \#A (related to composite A85-45b) and \#B (related to composite B100-45). 


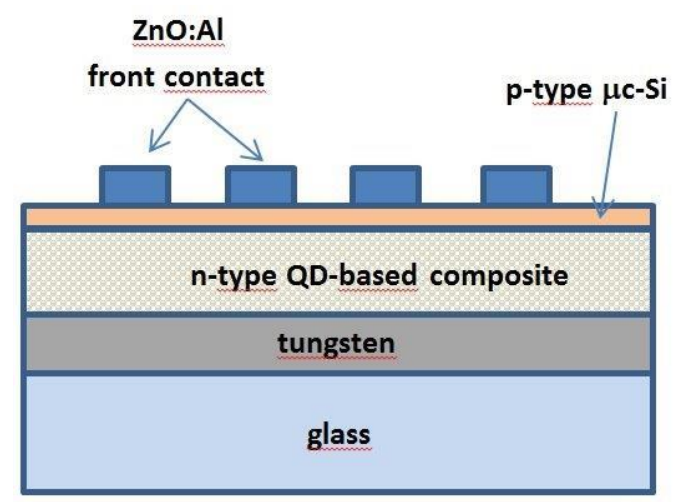

Figure 8 Representative scheme of PV p-n devices fabricated and characterized in this study.

In Figure 9, the dark and illuminated I-V measurements are reported for $\mathrm{p}-\mathrm{n}$ device \#A. The dark saturation current is $5 \times 10^{-6} \mathrm{~A} / \mathrm{cm}^{2}$, whereas the $V_{o c}$ and $J_{s c}$ values are $150 \mathrm{mV}$ and $0.055 \mathrm{~mA} / \mathrm{cm}^{2}$, respectively. In Figure 10, dark and illuminated I-V measurements are reported for device \#B. The diode performance was worse than the previous device $\left(J \mathrm{O}=4 \times 10^{-5} \mathrm{~A} / \mathrm{cm}^{2}\right)$. The $\mathrm{V}_{\text {oc }}$ and $\mathrm{J}_{\mathrm{sc}}$ values are $96 \mathrm{mV}$ and $0.47 \mathrm{~mA} / \mathrm{cm}^{2}$, respectively. There is an expected worsening of the $V_{\text {oc }}$ value (as a consequence of a too high Jo value) but, at the same time, there is an appreciable increase in the $\mathrm{J}_{\mathrm{sc}}$ value, one order larger than the previous device. This considerable increase can be explained in terms of the increased photoactive material thickness $(0.57 \mu \mathrm{m}$ vs. $0.28 \mu \mathrm{m})$.
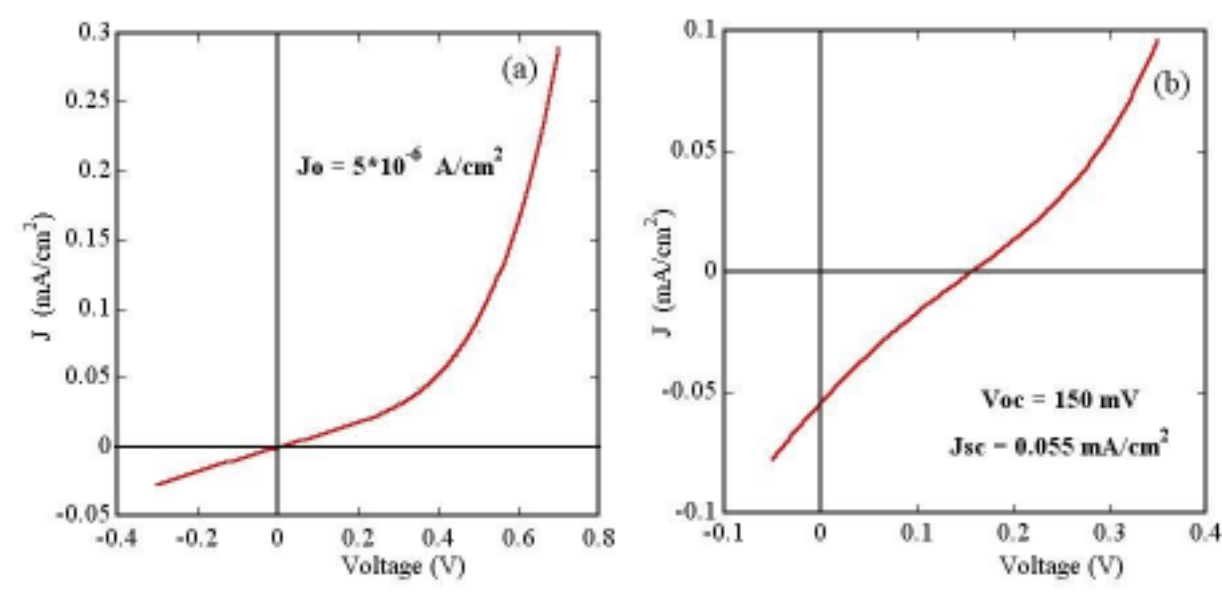

Figure 9 (a) Dark and (b) illuminated I-V measurements on $\mathrm{p}-\mathrm{n}$ device \#A.

Moreover, by taking into account the other illuminated device parameters, there is a large difference between the series resistance values of the two different devices, which are $100 \Omega \mathrm{cm}^{2}$ for device \#B and $3300 \Omega \mathrm{cm}^{2}$ for device \#A. At this point, it is necessary to remember that the two composites, viz., A85-45 and B100-45, showed different characteristics in terms of optical behaviors (see Table 1 in the previous paragraph), indicating different phases (zinc silicate, nano-amorphous $\mathrm{Si}$, nano-crystalline $\mathrm{Si}$, nanolayered $\mathrm{ZnO}$ ), different amounts for single-phase and different dimensions of nanostructures. These different compositional characteristics strongly influence both the number of the photo-generated hole-electron pairs and the diffusion length of the charge 
carriers, justifying the large difference in the collection of the photo-generated currents for the above-reported two devices.
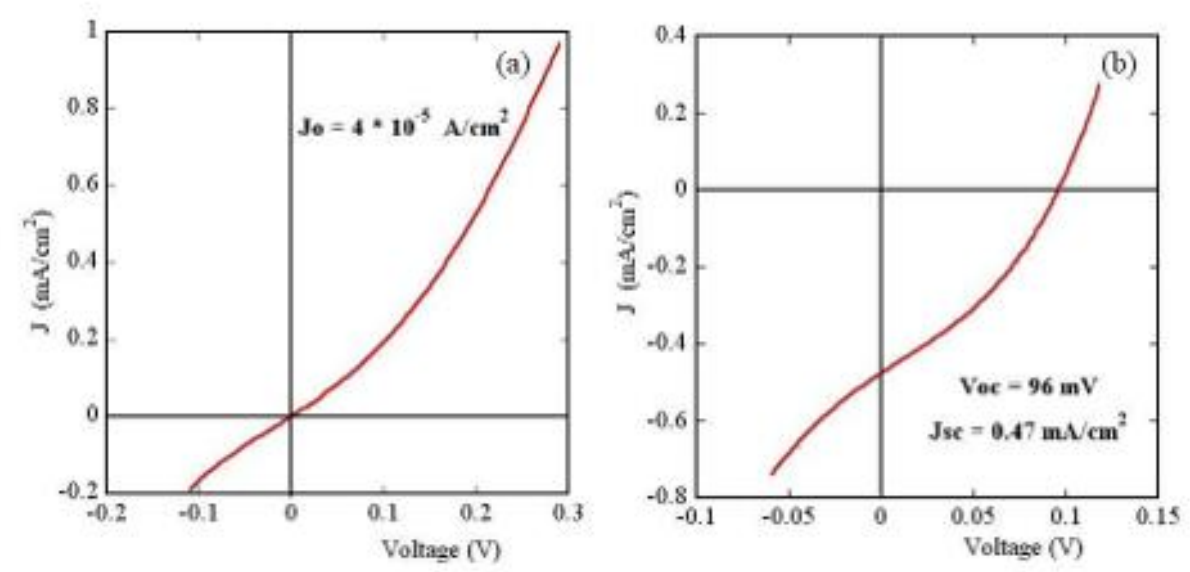

Figure 10 (a) Dark and (b) illuminated I-V measurements on p-n device \#B.

For both devices, the dark saturation currents are high, and the $\mathrm{V}_{\text {oc }}$ values are low $(150 \mathrm{mV}$ and $96 \mathrm{mV}$, respectively), and this could be attributed to an insufficient doping level inside composites. However, considering that the main aim of this experimental work is verifying the potentiality of photo-generating and collecting charge carriers, Quantum Efficiency measurements assume a strategic significance. In Figure 11, the QE spectra are reported for the two PV p-n devices. The integrated $\mathrm{J}_{\mathrm{sc}}$ values, 0.047 and $0.45 \mathrm{~mA} \mathrm{~cm}^{-2}$, are very similar to those obtained by illuminated I-V measurements. A more interesting aspect is represented by the spectral behaviors of the QE curves. In both graphs, we can see that the spectral response rises from zero only for photon energies higher than $2 \mathrm{eV}$, but it becomes very appreciable only for photon energies higher than $2.5 \mathrm{eV}$.
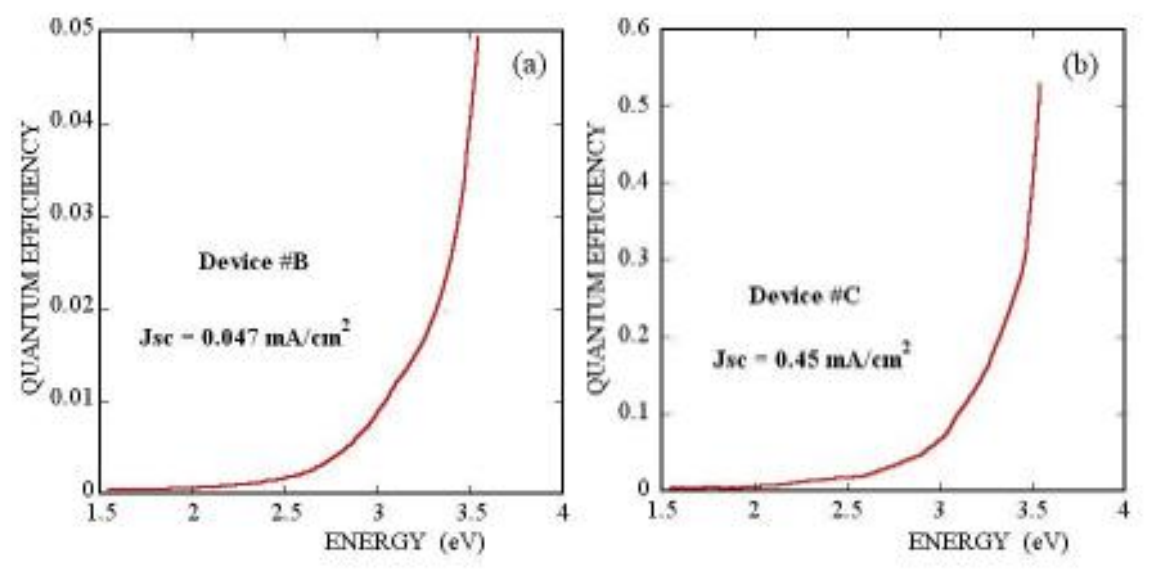

Figure 11 Quantum Efficiency measurements on p-n devices \#A and \#B.

The spectral behavior indicates two interesting aspects:

- In PV devices the charge carrier photo-generation can be exclusively attributed to silicon nanostructures. 
- The contribution of nano-crystalline Si to the photo-generated current is very poor, whereas the contribution of nano-amorphous $\mathrm{Si}$ is remarkable.

Regarding the PV p-n devices, the positive indications are (a) the feasibility of realizing PV devices with a very high optical gap ( $>2 \mathrm{eV}$ ) material, useful to fabricate high-performance multi-junction PV cells; (b) about device \#B, a remarkable collection of the photo-generated charge carriers in a $p$ $\mathrm{n}$ device (diffusion-type device) indicating a sufficiently large diffusion length of the minority carrier. At the same time, from a comparison with the data in the literature, there are two negative indications: (c) very poor quality $p-n$ diodes lead to very low $V_{o c}$; (d) photo-generating nanostructures are unbalanced toward too large energy threshold.

According to the above-mentioned considerations for obtaining higher charge carrier collection and, as a consequence, higher Jsc values, $p$-i-n devices (drift type) could be fabricated where the selected composites could be interposed between $\mathrm{p}$-Si and $\mathrm{n}$-Si layers deposited by PECVD, acting as i-layer. For improving spectral response in the range of $1.8-2.5 \mathrm{eV}$, a composite with a major amount of nc-Si can be utilized. However, the obtained results are promising, especially in consideration that the proposed low-temperature fabrication method utilized here is compatible with preserving underlying junction in an eventual multi-junction device.

\section{Conclusions}

In this work, we synthesized and characterized zinc silicate thin films, using a cheap and scalable solid-state reaction between sputtered reagents at significantly low temperatures ranging from 300 ${ }^{\circ} \mathrm{C}$ to $560^{\circ} \mathrm{C}$. Such new materials are amorphous, transparent in the visible region, chemically stable, with a high refractive index and ultralow dielectric constants. Moreover, inside such an inorganic guest host matrix, depending on reaction parameters, it is possible to host other phases like Sinanostructures and/or ZnO bulk and/or nano-crystalline, in amounts that can be properly tailored for specific optoelectronic applications. The presence of guests determines that from an insulating nature the zinc silicate composites become conductive, owing to the presence of thin islands of naturally $n$-doped bulk zinc oxide. As an example, if the relatively low temperature-based process is addressed to maximize nc-Si content, preserving ultrathin zinc oxide layers with efficient charge carrier transport can be obtained. In which, both are possible: the contribution coming from tunneling between $\mathrm{nc}-\mathrm{Si}$ and carrier motion through naturally $\mathrm{n}$-type zinc oxide islands. Tunable electrical behavior combined with visible transmittance is promising properties in fabricating photovoltaic junctions embeddable in multi-junction devices.

\section{Acknowledgments}

In this section, we would like to acknowledge Dr Alessandro Antonaia for introducing us into this research topic and helping us with Figure 4, before its retirement. We acknowledge lurie Usatie, too for CVD deposition of $p$-layer in cell devices.

\section{Author Contributions}

AC performed the synthesis and characterization of samples, GV performed technical support to samples annealing, EG performed ellipsometric and optical characterization. Authors joinly prepared the manuscript. 


\section{Competing Interests}

The authors have declared that no competing interests exist.

\section{References}

1. Liu Y, Shen J, Zhao L, Wang W, Gong W, Zheng F. Zinc-iron silicate for heterogeneous catalytic ozonation of acrylic acid: Efficiency and mechanism. RSC Adv. 2020; 10: 9146-9154.

2. El Mir L. Elaboration and characterization of glass-ceramic enriched by heavily manganese doped zinc silicate nanoparticles for optoelectronic applications. J Mater Sci Mater Electron. 2018; 29: 20493-20499.

3. Bharti DK, Gupta MK, Srivastava AK. Temperature dependent dielectric and electric properties of zinc silicate nanorods. Nano Struct Nano Objects. 2019; 17: 123-128.

4. Zaitseva NA, Onufrieva TA, Barykina JA, Krasnenko TI, Zabolotskaya EV, Samigullina RF. Magnetic properties and oxidation states of manganese ions in doped phosphor $\mathrm{Zn}_{2} \mathrm{SiO}_{4}$ : $\mathrm{Mn}$. Mater Chem Phys. 2018; 209: 107-111.

5. Castaldo A, Antonaia A, Addonizio ML. Synthesis of silicon quantum dots in zinc silicate matrix by low-temperature process: Optical, structural and electrical characterization. Thin Solid Films. 2014; 562: 172-180.

6. Bharti DK, Gupta MK, Kumar R, Sathish N, Srivastava AK. Non-centrosymmetric zinc silicategraphene based transparent flexible piezoelectric nanogenerator. Nano Energy. 2020; 73: 104821.

7. Mohd Shofri MF, Mohd Zaid MH, Matori KA, Fen YW, Yaakob Y, Jaafar SH, et al. Phase transformation, optical and emission performance of zinc silicate glass-ceramics phosphor derived from the $\mathrm{ZnO}-\mathrm{B}_{2} \mathrm{O}_{3}$-SLS glass system. Appl Sci. 2020; 10: 4940-4951.

8. Karazhanov SZ, Ravindran P, Fjellvåg H, Svensson BG. Electronic structure and optical properties of $\mathrm{ZnSiO}_{3}$ and $\mathrm{Zn}_{2} \mathrm{SiO}_{4}$. J Appl Phys. 2009; 106: 123701.

9. Ohtake T, Ohkawa K, Sonoyama N, Sakata T. A novel synthesis of $\mathrm{Zn}_{2} \mathrm{SiO}_{4}$ thin film on n-type $\mathrm{ZnO}$ semiconductor electrode and its electrochemical luminescence under the anodic polarization. J Alloys Compd. 2006; 421: 163-165.

10. Li X, Chen F. Structure and luminescence properties of $\mathrm{Zn}_{2} \mathrm{SiO}_{4}$ : Mn phosphor prepared with MCM-48. Mater Res Bull. 2013; 48: 2304-2307.

11. Nam SH, Kim MH, Lee JY, Lee SD, Boo JH. Spray pyrolysis of manganese doped zinc silicate phosphor particles. Funct Mater Lett. 2010; 3: 97-100.

12. Babu BC, Buddhudu S. Dielectric properties of willemite $\mathrm{Zn}_{2} \mathrm{SiO}_{4}$ nano powders by sol-gel method. Phys Procedia. 2013; 49: 128-136.

13. Castaldo A, Addonizio ML, Antonaia A, Nicotra G. A new route for fabrication of silicon QDs in a dielectric matrix of silica and silicate. Phys. Status Solidi C. 2012; 9: 1900-1903.

14. Sarikov A, Lisovskyy I. Spinodal decomposition versus nucleation and growth mechanism of phase separation in nonstoichiometric silicon oxide films during high temperature annealing. Solid State Commun. 2019; 287: 19-22.

15. Conibeer G, Perez-Wurfl I, Hao X, Di D, Lin D. Si solid-state quantum dot-based materials for tandem solar cells. Nanoscale Res Lett. 2012; 7: 193.

16. Coleman TF, Li Y. An interior trust region approach for nonlinear minimization subject to bounds. SIAM J Optim. 1996; 6: 418-445. 
17. Coleman TF, Li Y. On the convergence of interior-reflective Newton methods for nonlinear minimization subject to bounds. Math Program. 1994; 67: 189-224.

18. Kajiyama K, Yoneda T, Fujioka Y, Kido Y. Si O bond formation by oxygen implantation into silicon. Nucl Instrum Methods Phys Res B. 1997; 121: 315-318.

19. Pomjaturad J, Rattanakawin C, Sriprang N, Tongteeka S, Chairuangsri T. Effects of additives in froth flotation of silicate zinc ore; a study by zeta potential measurement and infrared spectroscopy. Chiang Mai J Sci. 2007; 34: 191-200.

20. Iacona F, Bongiorno C, Spinella C, Boninelli S, Priolo F. Formation and evolution of luminescent Si nanoclusters produced by thermal annealing of SiOx films. J Appl Phys. 2004; 95: 3723-3732.

21. Conibeer G, Green M, Cho EC, König D, Cho YH, Fangsuwannarak T, et al. Silicon quantum dot nanostructures for tandem photovoltaic cells. Thin Solid Films. 2008; 516: 6748-6756.

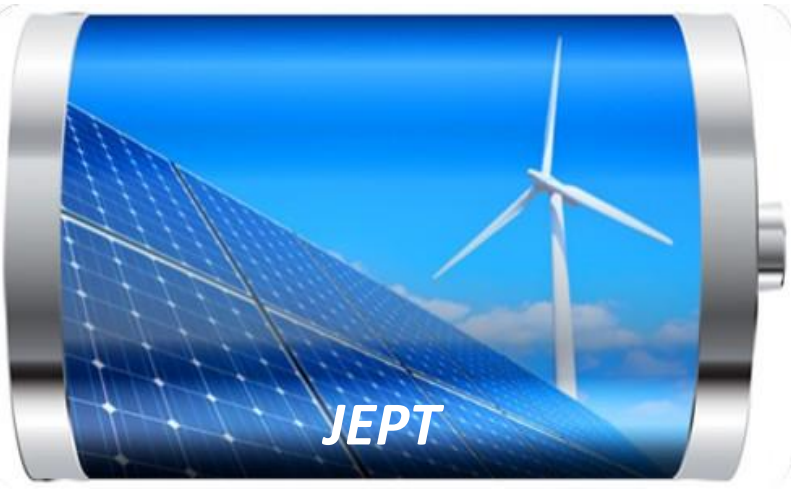

Enjoy JEPT by:

1. Submitting a manuscript

2. Joining in volunteer reviewer bank

3. Joining Editorial Board

4. Guest editing a special issue

For more details, please visit: http://www.lidsen.com/journal/jept 